\title{
Relationship between Molecular Weight and Viscosity for Polydispersed Poly(n-docosyl acrylate)
}

\author{
Shashi D. BARUAH ${ }^{\dagger}$ and Narayan C. LASKAR \\ Regional Research Laboratory, Jorhat 785006, Assam, India
}

(Received April 5, 1996)

\begin{abstract}
The Mark-Houwink-Sakurada (MHS) relation for polydispersed poly( $n$-docosyl acrylate) was established from gel permeation chromatography (GPC) and intrinsic viscosity measurements. Average $K$ and $\alpha$ for polydispersed comb-like poly $\left(n\right.$-docosyl acrylate) in tetrahydrofuran at $35^{\circ} \mathrm{C}$, were $4.95 \times 10^{-5} \mathrm{dl} \mathrm{g}^{-1}$ and 0.738 , respectively. The X-ray diffraction peak of poly $\left(n\right.$-docosyl acrylate) at about $2 \theta=22^{\circ}$ corresponded to the typical spacing of hexagonal packing of long alkyl side chains of the acrylate polymer.

KEY WORDS Poly(n-docosyl acrylate) / Comb-Like Polymer / Hydrodynamic Average Molecular Weight / Mark-Houwink-Sakurada Relation / Intrinsic Viscosity /
\end{abstract}

In a previous paper, ${ }^{1}$ the kinetics of high conversion polymerization of $n$-docosyl acrylate were reported. A linear polymer with pendent alkyl side chain of specific length is one of the general structural characteristics of flow improve (FI)/pour point depressant (PPD) for crude oils. A number of patents describe the use of polymers of higher alkyl acrylates and methacrylates as PPDs and/or FIs. ${ }^{2,3}$ We recently investigated the polymerization behavior of higher alkyl acrylates or methacrylates $^{1,4,5}$ to derive their structure reactivity relationship.

Poly (n-docosyl acrylate) $\left(\mathrm{PC}_{22} \mathrm{~A}\right)$ may be considered a typical short branched comb-like polymer because each has one long alkyl side chain on every two main chain carbon atoms. Studies on the dilute solution properties of narrowly spaced comb-like polymers are very few whereas the synthesis and side chain crystallization behaviour of various widely spaced comb-like polymers are studied in detail. ${ }^{6-7}$ The long alkyl side chain of the comb-like polymer tends to aggregate in solution and therefore exists in a less expanded form than polystyrene of the same molecular weight. ${ }^{8,9}$

Molecular weights of higher alkyl acrylate or methacrylate polymers determined by gel permeation chromatography (GPC) are reported with respect to the universal calibration curve. ${ }^{10}$ This might lead to a reasonable estimate of molecular weights, but the true molecular weight can be obtained only when the mathematical relationship describing the hydrodynamic volume of the calibration standard and polymer under investigation is known. ${ }^{10}$ This requires that Mark-HouwinkSakurada (MHS) constants of the long chain alkyl acrylate or methacrylate polymers are known.

MHS constants used to describe the viscosity-molecular weight relationship have traditionally been obtained for unknown polymers by the laboratory procedure of fractionating a broad molecular weight distribution sample and then measuring the molecular weight and intrinsic viscosity of each fraction by osmometry or light scattering. GPC can also be used for the determination of MHS constants for unfractionated polymer samples of known $\bar{M}_{n}$ and $\bar{M}_{w}$ and intrinsic viscosity.

\footnotetext{
† To whom correspondence should be addressed.
}

Recently Dobbin et al., ${ }^{11,12}$ following the work of Weiss and Cohn-Ginsberg ${ }^{13}$ reported a method by which MHS constants for a broad distribution polymer may be estimated from GPC and intrinsic viscosity data. Goldwasser et $a l .{ }^{14}$ defined a new average molecular weight, $\bar{M}_{x}$, which can also provide an alternate route to the determination of $K$ and $\alpha$ of polymers with broad molecular weight distribution (MWD) using GPC and intrinsic viscosity measurements. Mahabadi and Alexandru ${ }^{15}$ proposed a new and simple method for determination of MHS constants using hydrodynamic average molecular weight, $\bar{M}_{x}$, together with intrinsic viscosity data of several broad molecular weight distributed samples.

The objective of the present work is to establish the viscosity-molecular weight relationship from the hydrodynamic average molecular weight, $\bar{M}_{x}$, and intrinsic viscocity data of broad distributed $\mathrm{PC}_{22} \mathrm{~A}$ in tetrahydrofuran (THF) at $35^{\circ} \mathrm{C}$.

\section{EXPERIMENTAL}

The purification of $n$-docosyl acrylate monomer (Sidobre-Sinnova) was reported in an earlier communication. ${ }^{1}$ Poly( $n$-docosyl acrylate) was prepared using a free radical initiator in benzene. ${ }^{1}$ The polymers were purified by repeated precipitation in acetone.

Gel permeation chromatography (GPC) was performed using a Waters model 510 solvent delivery system at a flow rate of $1.0 \mathrm{~mL} \mathrm{~min}^{-1}$ through a set of four ultrastyragel columns (Waters) of exclusion sizes $10^{6}$, $10^{5}, 10^{4}$, and $500 \AA$. Analysis was performed at room temperature, using purified high-performance liquid chromatography (HPLC) grade THF as eluent. A differential refractometer model R401 from Waters was used as the detector. The volume of polymer injected was $50 \mu \mathrm{L}$. GPC curves were analyzed with the universal calibration curve obtained by 9 narrow-MWD polystyrene samples.

The X-ray diffractogram of $\mathrm{PC}_{22} \mathrm{~A}$ was recorded on a model JDX-11P3A JEOL diffractometer with a solid sample using Ni filter with $\mathrm{Cu}-K_{\alpha}$ radiation at $35 \mathrm{kV}$ and $10 \mathrm{~mA}$ in the wide angle range of $2^{\circ}<2 \theta<60^{\circ}$.

Intrinsic viscosities of polymer solutions were deter- 
mined at $35^{\circ} \mathrm{C}$ using a Schott Gerate model AVS 400 automated viscometer in different solvents. The temperature of the viscometer was maintained at $35 \pm 0.01^{\circ} \mathrm{C}$ using a constant temperature bath. Intrinsic viscosities were calculated by the single point method described by Solomon and Ciuta ${ }^{16}$ :

$$
[\eta]=\left[2\left(\eta_{\mathrm{sp}}-\ln \eta_{\mathrm{rel}}\right)\right]^{1 / 2} / C
$$

where $\eta_{\mathrm{sp}}$ and $\eta_{\mathrm{rel}}$ are the specific and relative viscosities of the polymer solution, respectively, and $\mathrm{C}$ is the concentration $\left(\mathrm{g} \mathrm{l}^{-1}\right)$. Equation 1 yields accurate intrinsic viscosities in the high reproducibility when measurements are made for dilute polymer solutions $(0.5 \% \mathrm{w} / \mathrm{v})$.

\section{RESULTS AND DISCUSSION}

Goldwasser et al. ${ }^{14}$ defined a new molecular weight average, $\bar{M}_{x}$ to characterize polymers of different composition in terms of the hydrodynamic volume:

$$
\begin{aligned}
\bar{M}_{x} & =\sum w_{\mathrm{i}}[\eta]_{\mathrm{i}} M_{\mathrm{i}} / \sum w_{\mathrm{i}}[\eta]_{\mathrm{i}} \\
& =\sum w_{\mathrm{i}} J_{\mathrm{i}} /[\eta]
\end{aligned}
$$

where $w_{\mathrm{i}}$ and $[\eta]_{\mathrm{i}}$ are the weight fraction and intrinsic viscosity of each fraction at elution volume $v_{\mathrm{i}}$ in the GPC system, respectively. The denominator in eq 2 is equal to the intrinsic viscosity $[\eta]$, of the whoel sample in GPC solvent. The value of the numerator is calculated from the GPC chromatogram with universal calibration. $J_{\mathrm{i}}=[\eta]_{\mathrm{i}} M_{\mathrm{i}}$ can be equated to the ratio of the area of the GPC detector response at elution volume $v_{\mathrm{i}}$ to the total area under the GPC chromatogram.

For a polydispersed polymer, the MHS equation relates $[\eta]$ to the viscosity average molecular weight, $\bar{M}_{v}$ :

$$
[\eta]=K \bar{M}_{v}^{\alpha}
$$

Mahabadi and Alexandru ${ }^{15}$ defined the MHS equation in terms of $\bar{M}_{x}$ and showed that,

$$
[\eta]=k_{x} \bar{M}_{x}^{\alpha}=\delta K \bar{M}_{x}^{\alpha}
$$

where $\delta$ is given by

$$
\delta=\left(\sum w_{\mathrm{i}} J_{\mathrm{i}}^{[\alpha /(1+\alpha)]}\right)^{(1+\alpha)} /\left(\sum w_{\mathrm{i}} J_{\mathrm{i}}\right)^{\alpha}
$$

From eq 5, for several samples of varying molecular weight, a $\ln -\ln$ plot of $[\eta]$ vs $\bar{M}_{x}$ should have a linear slope assuming $\delta$ remains constant. The constant $K=k_{x} / \delta$ can be calculated using eq 6 .

For polymers with the same polydispersity or having broad MWD, $\delta$, remains constant.

The hydrodynamic average molecular weight, $\bar{M}_{x}$, of $\mathrm{PC}_{22} \mathrm{~A}$ was determined in THF using eq 3 . The numerator of right hand side of the equation was evaluated from normalized GPC chromatogram data. Calculated $\bar{M}_{x}$ along with the experimental $[\eta]$ were applied to eq 5 for the determination of $k_{x}$ and $\alpha$ from least square analysis. A $\ln -\ln$ plot of $[\eta] v s . \bar{M}_{x}$ for $\mathrm{PC}_{22} \mathrm{~A}$ is shown in Figure 1, and $\alpha$ is estimated by the slope. Using the GPC chromatogram and estimated $\alpha, \delta$, and subsequently $K$ for each sample were determined. The different values of $\bar{M}_{x},[\eta], \delta$, and $K$ are summarized in Table I. The average values of $k_{x}, \alpha$, and $K$ were estimated as $2.72 \times 10^{-5} \mathrm{dlg}^{-1}, 0.738$, and $4.90 \times 10^{-5} \mathrm{dl} \mathrm{g}^{-1}$, respectively. The calculated values can be used to estab-

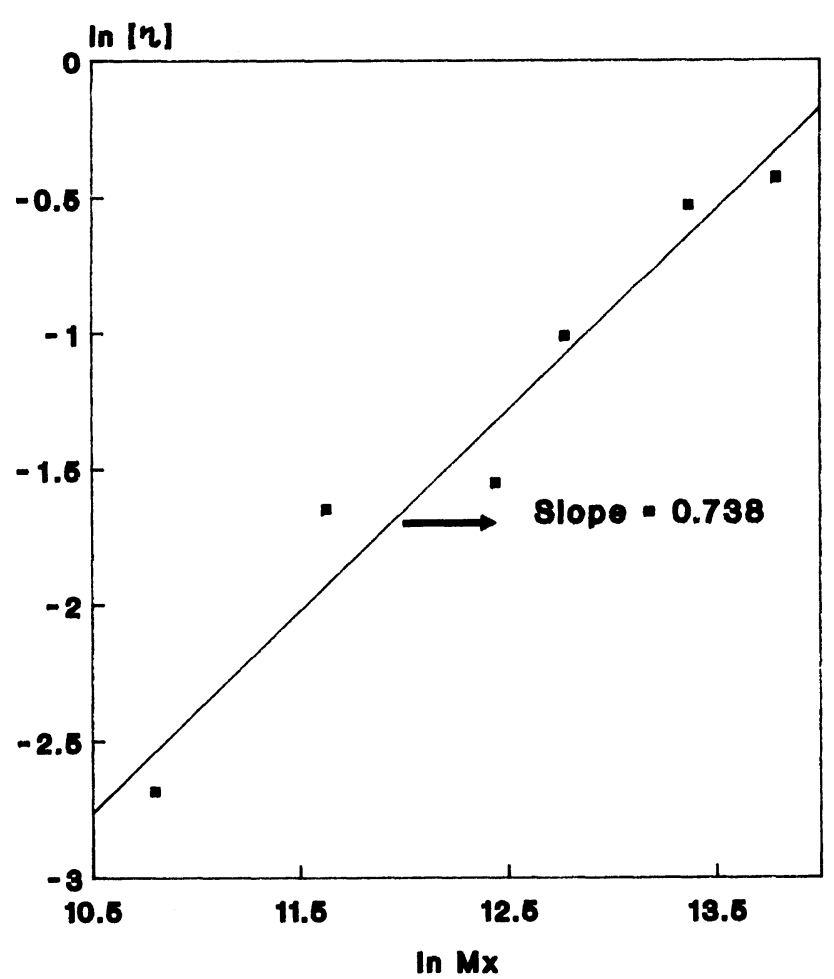

Figure 1. Plot of $\ln [\eta]$ vs. $\ln \bar{M}_{x}$ for poly $(n$-docosyl acrylate) in tetrahydrofuran (THF) at $35^{\circ} \mathrm{C}\left(\bar{M}_{w} / \bar{M}_{n}>2.5\right)$.

Table I. Intrinsic viscosity $-\bar{M}_{x}$ data for $\operatorname{poly}(n$-docosyl acrylate) of broad molecular weight distribution in tetrahydrofuran (THF) at $35^{\circ} \mathrm{C}$

\begin{tabular}{ccccc}
\hline$M_{w} / \bar{M}_{n}$ & $10^{-5} \times \bar{M}_{x}$ & {$[\eta] / \mathrm{dlg}^{-1}$} & $\delta^{\mathrm{a}}$ & $10^{-5} \times K^{\mathrm{b}} / \mathrm{dl} \mathrm{g}^{-1}$ \\
\hline 4.0 & 9.72 & 0.649 & 0.540 & 5.03 \\
5.6 & 6.40 & 0.587 & 0.575 & 4.73 \\
4.8 & 3.53 & 0.362 & 0.502 & 5.42 \\
3.3 & 2.52 & 0.211 & 0.553 & 4.92 \\
3.3 & 1.12 & 0.192 & 0.552 & 4.93 \\
2.6 & 0.49 & 0.068 & 0.582 & 4.67 \\
& & & & $\bar{K}=4.95 \times 10^{-5}$ \\
\hline
\end{tabular}

a $\delta$ calculated from eq 6 using $\alpha=0.738 .{ }^{\mathrm{b}} K$ calculated from $k_{x} / \delta$ using $k_{x_{(}}$(av) $=2.72 \times 10^{-5} \mathrm{dlg}^{-1}$.

Table II. Intrinsic viscosities of poly( $n$-docosyl acrylate) in different solvents at $35^{\circ} \mathrm{C}$

\begin{tabular}{cccccc}
\hline & & \multicolumn{4}{c}{ Intrinsic viscosities $[\eta] / \mathrm{dlg}^{-1}$} \\
\cline { 3 - 6 } $10^{-4} \times \bar{M}_{n}$ & $\bar{M}_{w} / \bar{M}_{n}$ & Xylene & Toluene & Benzene & THF \\
\hline 26.8 & & & & & \\
20.3 & 5.6 & 0.820 & 0.772 & 0.747 & 0.649 \\
10.3 & 4.8 & 0.728 & 0.698 & 0.659 & 0.587 \\
6.3 & 3.3 & 0.273 & 0.408 & 0.377 & 0.362 \\
4.8 & 3.3 & 0.232 & 0.218 & 0.227 & 0.211 \\
2.6 & 2.6 & 0.091 & 0.083 & 0.078 & 0.192 \\
& & & & & \\
\hline
\end{tabular}

lish the relationship between $[\eta]$ and molecular weight of polydispersed $\mathrm{PC}_{22} \mathrm{~A}$.

Intrinsic viscosities of $\mathrm{PC}_{22} \mathrm{~A}$ in different solvents are presented in Table II. This can be seen as additional verification of molecular weight determination. The sequence of $[\eta]$ in different solvents are $[\eta]_{\text {xylene }}>$ $[\eta]_{\text {toluene }}>[\eta]_{\text {benzene }}>[\eta]_{\mathrm{THF}}$. Intrinsic viscosities can be measured more accurately than averaged molecular 


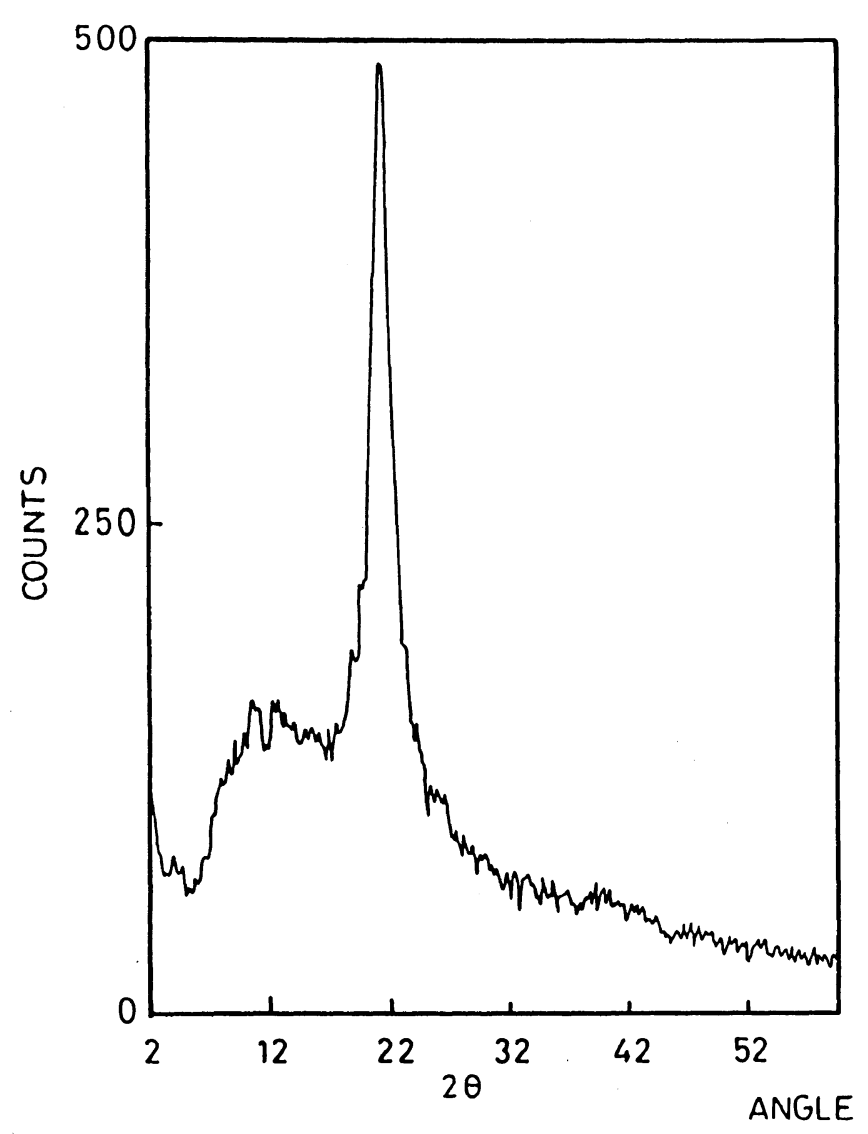

Figure 2. X-ray diffraction pattern of $\operatorname{poly}(n$-docosyl acrylate).

weights like $\bar{M}_{n}$ and $\bar{M}_{w}$.

This work shows that the method of Mahabadi and Alexandru leads to good estimation of MHS constants from GPC and intrinsic viscosity data for $\mathrm{PC}_{22} \mathrm{~A}$ and the averaging of a number of samples lead to more accurate estimation of molecular weights than the normal process which involves regression analysis of intrinsic viscosities of well characterized fractionated low dispersed polymer samples. $K$ and $\alpha$ determined in this work are valid for GPC measurements and molecular weight distribution of polydispersed $\mathrm{PC}_{22} \mathrm{~A}$.
The X-ray diffraction pattern of $\mathrm{PC}_{22} \mathrm{~A}$ (Figure 2) shows a peak at about $2 \theta=22^{\circ} \mathrm{C}$, which corresponds to a $d$ value of $4.16 \AA$. This corresponds to the value of several long chain acrylate and methacrylate comb-like polymers, ${ }^{8}$ attributed to the Van der Walls contact of non bonded atoms. ${ }^{17}$ Besides this peak, a very sharp peak at a very low angle (between $2-3^{\circ}$ ) is apparent. This indicates the presence of structures other than atomic contact structures ${ }^{17}$ in polydispersed comb-like $\mathrm{PC}_{22} \mathrm{~A}$.

Acknowledgment. The authors wish to thank the Director, RRL-Jorhat, for permission to publish these results.

\section{REFERENCES}

1. S. D. Baruah, N. C. Laskar, and B. Subrahmanyam, J. Appl. Polym. Sci., 51, 1701 (1994).

2. K. D. Miller and L. C. Parker, U.S. Patent, 3,675,671 (1972).

3. Exxon Research and Engineering Co., Ger. Patent, 2,613,315 (1976).

4. B. Subrahmanyam, S. D. Baruah, M. Rahman, J. N. Baruah, and N. N. Dass, J. Polym. Sci., Polym. Chem., 30, 2531 (1992).

5. B. Subrahmanyam, S. D. Baruah, M. Rahman, N. C. Laskar, and R. K. Mazumder, Polymer, 35, 862 (1994).

6. K. Yokota, M. Ohtubo, T. Hirabayashi, and Y. Inai, Polym. J., 25, 1079 (1993)

7. T. Hirabayashi, K. Kasabou, and K. Yokota, Polym. J., 20, 911 (1988).

8. K. Yokota and T. Hirabayashi, Polym. J., 18, 117 (1986).

9. K. Yokota, T. Hirabayashi, and Y. Inai, Polym. J., 26, 105 (1994).

10. Z. Grubisic, P. Rempp, and H. Benoit, J. Polym. Sci., B, 5, 753 (1967).

11. C. J. B. Dobbin, A. Rudin, and M. F. Tchir, J. Appl. Polym. Sci., 25, 2985 (1980).

12. C. J. B. Dobbin, A. Rudin, and M. F. Tchir, J. Appl. Polym. Sci., 27, 1081 (1982).

13. A. R. Weiss and E. Cohn-Ginsberg, J. Polym. Sci., Polym. Lett. Ed., 7, 379 (1969).

14. J. M. Goldwasser, A. Rudin, and W. L. Elsdon, J. Liquid Chromatogr., 5, 2253 (1982).

15. H. K. Mahabadi and L. Alexandru, Can. J. Chem., 63, 221 (1985).

16. O. F. Solomon and I. Z. Ciuta, J. Appl. Polym. Sci., 6, 683 (1962).

17. A. K. Chatterjee, S. D. Phatak, P. S. Murthy, and G. C. Joshi, J. Appl. Polym. Sci., 52, 887 (1994). 